\title{
Racial/Ethnic Differences in Diabetes Screening and Hyperglycemia Among US Women After Gestational Diabetes
}

\author{
Julie K. Bower, $\mathrm{PhD}^{1}$; Brittney N. Butler, MPH ${ }^{1,2}$; Seuli Bose-Brill, $\mathrm{MD}^{3}$; \\ Jennifer Kue, $\mathrm{PhD}^{4}$; Christina L. Wassel, $\mathrm{PhD}^{5}$
}

\begin{abstract}
Accessible Version: www.cdc.gov/pcd/issues/2019/19_0144.htm
Suggested citation for this article: Bower JK, Butler BN, BoseBrill S, Kue J, Wassel CL. Racial/Ethnic Differences in Diabetes Screening and Hyperglycemia Among US Women After Gestational Diabetes. Prev Chronic Dis 2019;16:190144. DOI: https://doi.org/10.5888/pcd16.190144.
\end{abstract}

\section{PEER REVIEWED}

\section{Summary}

What is already known on this topic?

Racial/ethnic minority women are disproportionately affected by type 2 diabetes. Among racial/ethnic minority women, non-Hispanic black women are at lowest risk for gestational diabetes mellitus (GDM) but highest risk for conversion to type 2 diabetes after GDM.

\section{What is added by this report?}

In this nationally representative population of US women with a history of GDM, non-Hispanic black women, compared with other racial/ethnic groups, were most likely to report receiving diabetes screening services in the past 3 years. However, non-Hispanic black and Hispanic women had higher $\mathrm{HbA}_{1 \mathrm{c}}$ levels and were more likely to have measured $\mathrm{HbA}_{1 \mathrm{c}}$ values in the prediabetes or diabetes range.

What are the implications for public health practice?

Screening for and prompt diagnosis of type 2 diabetes after GDM is critical in reducing complications. Differential receipt of follow-up services by racial/ethnic minority women may exacerbate disparities in the prevalence of type 2 diabetes.

\section{Abstract}

\section{Introduction}

Gestational diabetes mellitus (GDM) is the most common complication of pregnancy and is associated with an increased risk for type 2 diabetes. Racial/ethnic minority populations are at a higher risk than non-Hispanic white populations of developing type 2 diabetes after GDM. The aim of this study was to describe racial/eth- nic differences in hyperglycemia and receipt of screening services in a nationally representative sample of women with a history of GDM.

\section{Methods}

Our sample included 765 women from the US National Health and Nutrition Examination Survey (2007-2016) with a history of GDM. We used logistic, multinomial, linear, and proportional hazards regression to evaluate racial/ethnic differences in development of diabetes after GDM, hyperglycemia (measured by $\mathrm{HbA}_{1 \mathrm{c}}$ ), and receipt of diabetes screening services.

\section{Results}

Non-Hispanic black women had 63\% higher risk and Hispanic women and "other" racial/ethnic women had more than double the risk for diabetes compared with non-Hispanic white women. Among women with a GDM history who did not receive a diagnosis of diabetes by the time of the study examination, both nonHispanic black women and Hispanic women were more likely than non-Hispanic white women to be in the prediabetes or diabetes range (measured $\mathrm{HbA}_{1 \mathrm{c}} \geq 5.7 \%$ ). However, non-Hispanic black women had 2.07 (95\% confidence interval, 1.29-3.81) times the odds of being screened for diabetes compared with non-Hispanic white women $(P=.02)$.

\section{Conclusion}

Delays in identification of hyperglycemia and diagnosis of diabetes in racial/ethnic minority women may reflect differential delivery of guideline-based care or poor follow-up of abnormal screening test results.

\section{Introduction}

Gestational diabetes mellitus (GDM) affects $2 \%$ to $14 \%$ of all pregnancies in the United States and confers a significant and lifelong health challenge for both mother and infant, including an 
increased risk for type 2 diabetes (1-3). Approximately $60 \%$ of women with GDM will develop diabetes within 10 years after delivery, and GDM history is associated with a 7-fold increased lifetime risk for diabetes $(2,3)$. This population comprises a high-risk group that should be targeted for enhanced diabetes screening.

Clinical guidelines recommend postpartum glucose screening 4 to 12 weeks after a GDM pregnancy, yet an inadequate proportion of women with GDM receive these screenings (4). Estimates for receipt of the postpartum glucose screening vary between one-third and three-quarters of postpartum women, depending on the population, with low-income and racial/ethnic minority populations less likely to meet guideline-based screening recommendations (5-7).

For people who do not receive a diagnosis of diabetes at the postpartum visit $(90 \%-95 \%$ of all those screened), clinical guidelines recommend enhanced rescreening (8). For people with postpartum glucose screening results in the normoglycemic range, rescreening is recommended at least every 3 years; people with values in the prediabetes range should be rescreened annually. This repeated screening approach improves timely identification of prediabetes or type 2 diabetes and increases success of efforts to prevent or delay progression to type 2 diabetes.

The likelihood of developing type 2 diabetes after GDM differs across racial/ethnic groups. For example, black women with GDM are nearly 10 times as likely as women of all racial/ethnic groups without GDM to develop type 2 diabetes (9). We hypothesized that these observed disparities may partially be driven by differential receipt of screening services. Thus, the aim of this study was to describe racial/ethnic differences in the development of type 2 diabetes, levels of glycated hemoglobin $\left(\mathrm{HbA}_{1 c}\right)$ (a measure of hyperglycemia), and receipt of screening services based on clinical guidelines in a nationally representative sample of women with a history of GDM.

\section{Methods}

We analyzed data from the National Health and Nutrition Examination Survey (NHANES) for 2007-2016. NHANES is a cross-sectional, nationally representative survey of the US civilian noninstitutionalized population conducted by the National Center for Health Statistics (10). This study was a secondary data analysis that included 765 NHANES participants with a history of GDM who self-identified as female, were aged 18 years or older, and had self-reported information on current diabetes status at the time of their NHANES examination. A human subjects review board approved data collection procedures, and written informed consent was obtained from all study participants (11).

\section{Measures}

Race/ethnicity and other demographic characteristics, GDM and diabetes diagnoses, current use of insulin, reproductive history, health behaviors, health history, family history, health insurance status, health care access, and receipt of diabetes screening tests were determined by self-report. We categorized race/ethnicity as non-Hispanic white, non-Hispanic black, Hispanic, and "other." The "other" category comprises women in all other racial/ethnic categories indicated in NHANES as well as women who indicated multiple races/ethnicities. Income-to-poverty ratio was quantified by dividing family income by the poverty threshold determined by the US Department of Health and Human Services, specific to family size; a poverty-income ratio below 1 indicates that the family is below the poverty threshold. GDM history was defined as self-reported diagnosis of diabetes for the first time during pregnancy; age at GDM diagnosis was also reported. Development of type 2 diabetes after GDM was defined at the time of NHANES examination as self-reported diagnosis of diabetes by a health care provider (not including diabetes diagnosed during pregnancy) or use of insulin; age at diabetes diagnosis was also reported by participants. For women who did not develop diabetes after GDM, receipt of screening for diabetes during the previous 3 years was determined by using the following question: "Have you had a blood test for high blood sugar or diabetes within the past three years?" Blood pressure, body mass index (BMI), waist circumference, and lipids were directly measured by using standard methods described previously (12). $\mathrm{HbA}_{1 \mathrm{c}}$ was measured by using high-performance liquid chromatography, standardized to the Diabetes Control and Complications Trial assay (13).

\section{Statistical analysis}

We assessed differences in participant characteristics across racial/ ethnic groups by analysis of variance or $\chi^{2}$ test as appropriate. For analyses, we defined undiagnosed diabetes as measured $\mathrm{HbA}_{1 \mathrm{c}}$ equal to or greater than $6.5 \%$ in the absence of a diagnosis of diabetes or use of insulin and prediabetes as $\mathrm{HbA}_{1 \mathrm{c}}$ from $5.7 \%$ to $6.4 \%$ in the absence of a diagnosis of diabetes or use of insulin. We used multinomial logistic regression to evaluate the association of race/ethnicity with undiagnosed diabetes $\left(\mathrm{HbA}_{1 \mathrm{c}} \geq 6.5 \%\right)$, prediabetes $\left(\mathrm{HbA}_{1 \mathrm{c}} 5.7 \%-6.4 \%\right)$, and no diabetes $\left(\mathrm{HbA}_{1 \mathrm{c}}<5.7 \%\right)$ at the time of NHANES examination among women with no diabetes (as defined by self-report diagnosis or use of insulin). We evaluated associations of race/ethnicity with $\mathrm{HbA}_{1 \mathrm{c}}$ as a continuous outcome at the time of NHANES examination by using multivariable linear regression, stratified by women with and without diabetes.

Cox proportional hazards regression was used to assess the association of race/ethnicity with development of type 2 diabetes after

\footnotetext{
The opinions expressed by authors contributing to this journal do not necessarily reflect the opinions of the U.S. Department of Health and Human Services, the Public Health Service, the Centers for Disease Control and Prevention, or the authors' affiliated institutions.
} 
GDM, using the difference between age at GDM diagnosis and age at diabetes diagnosis after pregnancy (if applicable) as the time to event; for participants who did not develop diabetes, we calculated follow-up time as the difference between GDM diagnosis and current age. Women were excluded if they reported an age at type 2 diabetes diagnosis that was before the age at GDM diagnosis or had missing information on age at type 2 diabetes diagnosis or GDM diagnosis. The proportional hazards assumption was assessed, and it was not violated.

Associations of race/ethnicity, socioeconomic status, and access to health care with receipt of screening services were modeled by using logistic regression among women without diabetes (based on self-report or insulin use information). Analyses incorporated the NHANES sample weights and accounted for the complex sample survey design by using standard methods (12). We used the Taylor series linearization method for variance estimation. Analyses were performed in Stata Statistical Software release 14.2 (StataCorp LLC).

\section{Results}

Among 765 women with a history of GDM, 24.4\% developed type 2 diabetes after GDM (weighted percentage). This did vary, although not significantly, across race/ethnicity and was highest among non-Hispanic black women $(30.8 \%)$ and Hispanic women $(31.0 \%)$ (Table 1). Approximately one-fifth (22.1\%) of non-Hispanic white women and $18.3 \%$ of women of "other" race/ethnicity developed type 2 diabetes after GDM (Table 1). Women differed significantly across racial/ethnic groups at the time of NHANES examination in the number of past pregnancies $(P<$ $.001)$, BMI $(P<.001)$, hypertension prevalence $(P=.015)$, and waist circumference $(P<.001)$ (Table 1$)$. Hispanic women were most likely to report not having health insurance $34.8 \%$ of Hispanic women, compared with $12.5 \%$ of non-Hispanic white women) $(P<.001)$, and $20.0 \%$ of Hispanic women reported not having access to a routine location for health care $(P=.009$ across racial/ethnic groups).

\section{Associations of race/ethnicity with $\mathrm{HbA}_{1 \mathrm{c}}$}

In a fully adjusted model (Table 2), non-Hispanic black women, Hispanic women, and women in "other" racial/ethnic groups had significantly higher $\mathrm{HbA}_{1 \mathrm{c}}$ levels than did non-Hispanic white women. Among women with diabetes (by self-report or insulin use), non-Hispanic black and Hispanic women had $1.32 \%(P=.002)$ and $1.31 \%(P<.001)$ higher $\mathrm{HbA}_{1 \mathrm{c}}$, respectively, compared with non-Hispanic white women; the "other" racial/ethnic group did not differ significantly from the non-Hispanic white group $(P=$ .57). Among women with no diabetes, non-Hispanic black and Hispanic women had $0.34 \%$ higher $\operatorname{HbA}_{1 \mathrm{c}}(P=.004$ among non-
Hispanic black women; $P=.001$ among Hispanic women), whereas the "other" racial/ethnic group had a $0.14 \%$ higher $\operatorname{HbA}_{1 \mathrm{c}}(P=$ $.03)$, compared with non-Hispanic white women. In fully adjusted models (Table 2), the relative risk ratio for prediabetes was 3.4fold higher (95\% confidence interval [CI], 1.60-7.04); $P=.002)$ and for $\mathrm{HbA}_{1 \mathrm{c}} \geq 6.5 \%$ was 5.2-fold higher (95\% CI, 1.39-19.70); $P$ $=.02$ ) among non-Hispanic black women than among non-Hispanic white women. Among Hispanic women, the relative risk ratio for prediabetes was 2.2-fold higher $(95 \% \mathrm{CI}, 1.17-4.17 ; P=.02)$ and for $\mathrm{HbA}_{1 \mathrm{c}} \geq 6.5 \%$ was 6.7 -fold higher (95\% CI, 2.51-17.98; $P$ $<.001)$ than among non-Hispanic white women.

\section{Associations of race/ethnicity with development of type 2 diabetes after GDM}

Among the 765 women with a history of GDM, 6 were missing information on age at diabetes diagnosis and were excluded from analysis. In fully adjusted models, non-Hispanic black women had a $63 \%$ higher risk $(95 \% \mathrm{CI}, 1.11-2.39 ; P=.01)$, Hispanic women more than double the risk (hazard ratio $=2.22 ; 95 \%$ CI, 1.47-3.35; $P<.001$ ), and "other" racial/ethnic women approximately double the risk (hazard ratio $=2.08 ; 95 \% \mathrm{CI}, 1.01-4.28 ; P=.047$ ) of developing type 2 diabetes following GDM compared with non-Hispanic whites (Table 3).

\section{Receipt of screening among women without diabetes}

Among women without diabetes (defined by self-report or insulin use) at the NHANES examination, 67.1\% reported having had a diabetes screening test at least once in the past 3 years. By race/ ethnicity, this percentage was $67.1 \%$ among non-Hispanic white women, $77.3 \%$ among non-Hispanic black women, $59.2 \%$ among Hispanic women, and $72.6 \%$ among women in "other" racial/ethnic groups (Table 1). In adjusted models, non-Hispanic black women had 2.07 times the odds of being screened for diabetes compared with non-Hispanic white women (95\% CI, 1.29-3.81; $P=$ .02) (Table 4). Women with a higher income-to-poverty ratio (odds ratio $[\mathrm{OR}]$ per 1 -unit increment $=1.27$; 95\% CI, 1.08-1.49; $P=.005)$ were more likely to have been screened, and women who reported no access to a routine place for health care (compared with those with access to $\geq 1$ location for regular health care) had 0.44 times the odds of being screened (95\% CI, 0.22-0.90; $P$ $=.02$ ) (Figure). Other potential factors were considered but were removed from the final model because of no observed association; these factors were education, marital status, current age, and health insurance status. Sensitivity analyses adjusting for clinical diabetes risk factors only slightly attenuated the findings on race/ethnicity.

The opinions expressed by authors contributing to this journal do not necessarily reflect the opinions of the U.S. Department of Health and Human Services, the Public Health Service, the Centers for Disease Control and Prevention, or the authors' affiliated institutions. 


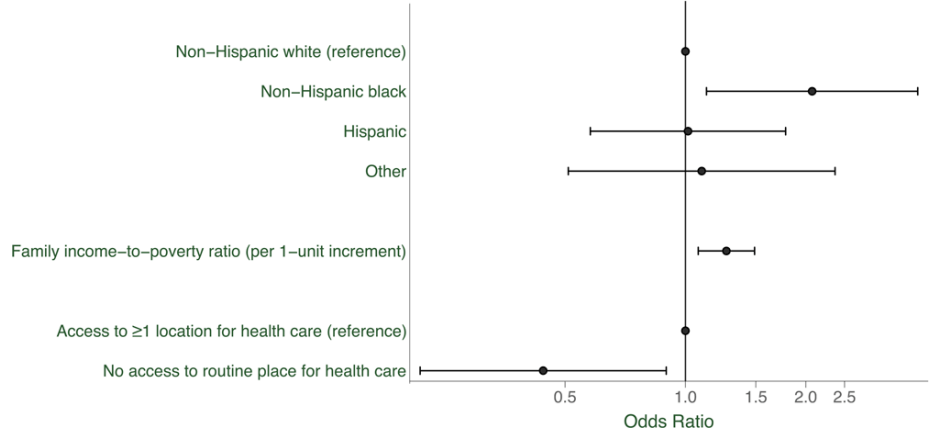

Figure. Racial/ethnic differences in having been screened for diabetes at least once in the past 3 years, US women without diabetes $(n=496)$. Odds ratios were adjusted for all other variables in the figure. Error bars indicate $95 \%$ confidence intervals.

\section{Discussion}

In our study, non-Hispanic black and Hispanic women were at higher risk for developing type 2 diabetes after GDM compared with non-Hispanic white women, and they had higher $\mathrm{HbA}_{1 \mathrm{c}}$ levels. Among all racial/ethnic groups examined, non-Hispanic black women were most likely to have measured $\mathrm{HbA}_{1 \mathrm{c}}$ values in the prediabetes $\left(\mathrm{HbA}_{1 \mathrm{c}} 5.7 \%-6.4 \%\right)$ and diabetes range $\left(\mathrm{HbA}_{1 \mathrm{c}} \geq\right.$ $6.5 \%$ in the absence of a diagnosis of diabetes). Both groups had higher rates of prediabetes and diabetes compared with non-Hispanic white women. Non-Hispanic black and Hispanic women had significantly higher mean $\mathrm{HbA}_{1 \mathrm{c}}$ levels, regardless of current diagnosed diabetes status.

Racial/ethnic disparities in risk for type 2 diabetes after GDM are well documented. Although non-Hispanic white and black women are at similar risk for GDM, black women have more than 2 times higher risk for developing type 2 diabetes after GDM compared with non-Hispanic white women (9). Heterogeneity in the burden of GDM risk and consequences within these subgroups cannot be fully explained by biological factors and traditional diabetes risk factors, and it is likely strongly influenced by social determinants and differential patterns of clinical practice (14). In our study, racial/ethnic differences in risk for diagnosed type 2 diabetes after GDM persisted even after adjustment for traditional diabetes risk factors such as age, education level, and BMI, and health insurance status. These findings demonstrate a gap in our understanding of individual and clinical practice factors - beyond traditional diabetes risk factors - influencing development of type 2 diabetes among racial/ethnic minority women.

The proportion of women reporting receipt of a diabetes screening test varied by race/ethnicity in the US population of women with a GDM history, despite the clinical recommendation that all women with GDM history be screened every 1 to 3 years. The finding that non-Hispanic black women without current diabetes, but with a history of GDM, were more likely to be screened for diabetes is consistent with their more adverse diabetes risk profile, including higher mean blood pressure, BMI, and prevalence of family history of diabetes. Despite being screened and demonstrating a higher prevalence of hyperglycemia, they were less likely to receive a diagnosis of diabetes. In unadjusted (crude) models, nonHispanic black women had higher odds and Hispanic women had lower odds of being screened compared with non-Hispanic white women. This association became nonsignificant after adjustment for sociodemographic, clinical, and health care access factors for Hispanic women, but the association became stronger for non-Hispanic black women.

The lower percentage of Hispanic women who reported being screened for diabetes is consistent with the observed lower percentage who had current health insurance and lower percentage reporting access to a routine location to obtain health care compared with non-Hispanic white women. More than $30 \%$ of Hispanic women with a GDM history were subsequently diagnosed with diabetes, higher than the percentage among non-Hispanic white women and similar to the percentage among non-Hispanic black women. However, among women that had not been diagnosed with diabetes, Hispanic women had lower odds of being screened for diabetes in the previous 3 years compared with all other racial/ethnic groups in unadjusted models. The association of race/ethnicity with receipt of screening became nonsignificant for Hispanic women after adjusting for measures of household income and access to a routine health care location. This finding suggests that access to screening services may play an important role in the observed lower likelihood of diabetes screening in this population.

Our findings also support research that non-Hispanic black women, in particular, are more likely to have undiagnosed prediabetes or diabetes and have a higher prevalence of suboptimal $\mathrm{HbA}_{1 \mathrm{c}}$ (15-19). These findings might partially reflect delays in early diagnosis of diabetes that may result from differential delivery of guideline-based care and/or follow-up of abnormal screening test results. Non-Hispanic black women may be screened more frequently because of perceived increased risk or higher prevalence of known diabetes risk factors, but evidence suggests that we are missing opportunities for early identification of hyperglycemia in this population. Health care providers play an important role in the diagnosis and treatment of diabetes for at-risk populations. Timely coordinated medical care is crucial because racial/ ethnic minority patients are often diagnosed at later stages of disease than are other racial/ethnic groups (20). Studies are needed to further examine racial/ethnic differences in diagnosis and treatment of diabetes, as well as the underlying factors that may limit

The opinions expressed by authors contributing to this journal do not necessarily reflect the opinions of the U.S. Department of Health and Human Services, the Public Health Service, the Centers for Disease Control and Prevention, or the authors' affiliated institutions. 
adherence to guideline-based care among racial/ethnic minority populations.

Although NHANES is a cross-sectional survey, it collects detailed information about pregnancy history and timing of diabetes diagnoses, allowing for estimation of diabetes risk after GDM. Additionally, non-Hispanic black and Hispanic women were oversampled to provide a large study population to evaluate racial/ethnic differences; Asian women were not oversampled in most survey years and, therefore, we were not able to look at this subpopulation separately. Finally, diabetes diagnosis information was collected via self-report; potential misclassification cannot be ruled out. However, the study is strengthened by inclusion of a nationally representative sample of US women with a GDM history and extensive information about their social and family history, health behaviors, health status and history, and several indicators related to access to health services.

Screening and diagnosis of GDM is critical in reducing complications in women and their infants, as well as preventing onset of type 2 diabetes. Differential receipt of follow-up services by racial/ethnic minority women may exacerbate observed disparities in the burden of type 2 diabetes. Our study showed racial/ethnic disparities in diabetes diagnosis, receipt of diabetes screening tests, and $\mathrm{HbA}_{1 \mathrm{c}}$ levels. Additional investigation to identify underlying factors contributing to this observed disparity will be particularly important to inform recommendations to ensure more equitable delivery of quality care across populations.

\section{Acknowledgments}

No financial support was received for this research. No copyrighted material, surveys, instruments, or tools were used in this article.

\section{Author Information}

Corresponding Author: Julie K. Bower, PhD, The Ohio State University College of Public Health, Division of Epidemiology, 1841 Neil Ave, Suite 300, Columbus, OH 43210. Telephone: 614247-8916. Email: Bower.185@osu.edu.

Author Affiliations: ${ }^{1}$ The Ohio State University College of Public Health, Columbus, Ohio. ${ }^{2}$ Kirwan Institute for the Study of Race and Ethnicity, The Ohio State University, Columbus, Ohio. ${ }^{3}$ The Ohio State University College of Medicine, Columbus, Ohio. ${ }^{4}$ The Ohio State University College of Nursing, Columbus, Ohio. ${ }^{5}$ Applied Sciences, Premier, Inc, Charlotte, North Carolina.

\section{References}

1.Ferrara A, Hedderson MM, Albright CL, Ehrlich SF, Quesenberry CP Jr, Peng T, et al. A pregnancy and postpartum lifestyle intervention in women with gestational diabetes mellitus reduces diabetes risk factors: a feasibility randomized control trial. Diabetes Care 2011;34(7):1519-25.

2. Bellamy L, Casas JP, Hingorani AD, Williams D. Type 2 diabetes mellitus after gestational diabetes: a systematic review and meta-analysis. Lancet 2009;373(9677):1773-9.

3. Centers for Disease Control and Prevention. National diabetes fact sheet: national estimates and general information in diabetes and prediabetes in the United States, 2011. Atlanta (GA): US Department of Health and Human Services, Centers for Disease Control and Prevention; 2011.

4. American Diabetes Association. Standards of medical care in diabetes - 2017: summary of revisions. Diabetes Care 2017; 40(Suppl 1):S4-5.

5. Tovar A, Chasan-Taber L, Eggleston E, Oken E. Postpartum screening for diabetes among women with a history of gestational diabetes mellitus. Prev Chronic Dis 2011; 8(6):A124.

6. Russell MA, Phipps MG, Olson CL, Welch HG, Carpenter MW. Rates of postpartum glucose testing after gestational diabetes mellitus. Obstet Gynecol 2006;108(6):1456-62.

7. Hale NL, Probst JC, Liu J, Martin AB, Bennett KJ, Glover S. Postpartum screening for diabetes among Medicaid-eligible South Carolina women with gestational diabetes. Womens Health Issues 2012;22(2):e163-9.

8. American Diabetes Association. 13. Management of diabetes in pregnancy: standards of medical care in diabetes - 2018 . Diabetes Care 2018;41(Suppl 1):S137-43.

9. Xiang AH, Li BH, Black MH, Sacks DA, Buchanan TA, Jacobsen SJ, et al. Racial and ethnic disparities in diabetes risk after gestational diabetes mellitus. Diabetologia 2011; 54(12):3016-21.

10. Centers for Disease Control and Prevention, National Center for Health Statistics. National Health and Nutrition Examination Survey. 2015. http://www.cdc.gov/NCHS/ nhanes.htm. Accessed July 11, 2015.

11. Centers for Disease Control and Prevention, National Center for Health Statistics. NCHS Research Ethics Review Board (ERB) approval. 2017. http://www.cdc.gov/nchs/nhanes/ irba98.htm. Accessed January 19, 2018.

12. Centers for Disease Control and Prevention, National Center for Health Statistics. National Health and Nutrition Examination Survey. 2018. http://www.cdc.gov/NCHS/ nhanes.htm. Accessed February 2, 2018.

The opinions expressed by authors contributing to this journal do not necessarily reflect the opinions of the U.S. Department of Health and Human Services, the Public Health Service, the Centers for Disease Control and Prevention, or the authors' affiliated institutions. 
13. Centers for Disease Control and Prevention, National Center for Health Statistics. Laboratory procedure manual. https:// wwwn.cdc.gov/nchs/data/nhanes/2015-2016/labmethods/ GHB_I_MET.pdf. Accessed October 1, 2019.

14. Black SA. Diabetes, diversity, and disparity: what do we do with the evidence? Am J Public Health 2002;92(4):543-8.

15. Harris MI, Eastman RC, Cowie CC, Flegal KM, Eberhardt MS. Racial and ethnic differences in glycemic control of adults with type 2 diabetes. Diabetes Care 1999;22(3):403-8.

16. Britton LE, Hussey JM, Crandell JL, Berry DC, Brooks JL, Bryant AG. Racial/ethnic disparities in diabetes diagnosis and glycemic control among women of reproductive age. J Womens Health (Larchmt) 2018;27(10):1271-7.

17. Cowie CC, Rust KF, Byrd-Holt DD, Gregg EW, Ford ES, Geiss LS, et al. Prevalence of diabetes and high risk for diabetes using hemoglobin A1c criteria in the US population in 1988-2006. Diabetes Care 2010;33(3):562-8.

18. Menke A, Casagrande S, Geiss L, Cowie CC. Prevalence of and trends in diabetes among adults in the United States, 1988-2012. JAMA 2015;314(10):1021-9.

19. Selvin E, Parrinello CM, Sacks DB, Coresh J. Trends in prevalence and control of diabetes in the United States, 1988-1994 and 1999-2010. Ann Intern Med 2014; 160(8):517-25.

20. Goonesekera SD, Yang MH, Hall SA, Fang SC, Piccolo RS, McKinlay JB. Racial ethnic differences in type 2 diabetes treatment patterns and glycaemic control in the Boston Area Community Health Survey. BMJ Open 2015;5(5):e007375.

The opinions expressed by authors contributing to this journal do not necessarily reflect the opinions of the U.S. Department of Health and Human Services, the Public Health Service, the Centers for Disease Control and Prevention, or the authors' affiliated institutions. 


\section{Tables}

Table 1. Characteristics of Women Aged $\geq 18$ Years With a History of Gestational Diabetes Mellitus $(\mathrm{N}=765)$, National Health and Nutrition Examination Survey (NHANES), 2007-2016 ${ }^{\mathrm{a}}$

\begin{tabular}{|c|c|c|c|c|c|}
\hline Characteristic & $\begin{array}{l}\text { Non-Hispanic White } \\
\quad(\mathrm{n}=267)\end{array}$ & $\begin{array}{l}\text { Non-Hispanic Black } \\
\quad(n=138)\end{array}$ & $\begin{array}{l}\text { Hispanic } \\
(n=266)\end{array}$ & $\begin{array}{l}\text { Other }^{\mathrm{b}} \\
(\mathrm{n}=94)\end{array}$ & $P$ Value $^{\mathrm{c}}$ \\
\hline Age, y & $46.5(0.9)$ & $44.4(1.0)$ & $41.8(0.7)$ & $42.6(1.6)$ & $<.001$ \\
\hline No. of live births & $2.4(0.1)$ & $2.7(0.1)$ & $3.0(0.1)$ & $2.0(0.2)$ & $<.001$ \\
\hline No. of pregnancies & $3.3(0.1)$ & $3.9(0.2)$ & $3.9(0.1)$ & $3.0(0.2)$ & $<.001$ \\
\hline Body mass index, $\mathrm{kg} / \mathrm{m}^{2}$ & $31.2(0.5)$ & $32.8(0.9)$ & $31.7(0.5)$ & $28.1(1.3)$ & $<.001$ \\
\hline Waist circumference, $\mathrm{cm}$ & $102.0(1.1)$ & $104.4(1.9)$ & $101.5(1.1)$ & $93.8(2.5)$ & .02 \\
\hline Current smoker, $\%$ & 19.3 & 19.6 & 10.8 & 9.1 & $<.001$ \\
\hline Systolic blood pressure, $\mathrm{mm} \mathrm{Hg}$ & $116.5(1.1)$ & $123.9(1.7)$ & $120.7(1.3)$ & $119.5(4.4)$ & .23 \\
\hline Diastolic blood pressure, $\mathrm{mm} \mathrm{Hg}$ & $70.9(0.7)$ & $71.9(1.1)$ & $68.9(1.0)$ & $69.8(1.6)$ & .02 \\
\hline Hypertension, \% & 37.5 & 56.1 & 34.1 & 34.5 & .02 \\
\hline Total cholesterol, mg/dL & $198.8(3.1)$ & $186.7(3.6)$ & $200.4(4.2)$ & $198.8(10.8)$ & .52 \\
\hline $\begin{array}{l}\text { High-density lipoprotein cholesterol, } \\
\mathrm{mg} / \mathrm{dL}\end{array}$ & $54.3(1.1)$ & $54.0(1.5)$ & $52.2(1.4)$ & $53.4(2.2)$ & .40 \\
\hline Family history of diabetes, $\%$ & 58.2 & 69.8 & 59.7 & 61.7 & .72 \\
\hline Developed type 2 diabetes after GDM, \% & 22.1 & 30.8 & 31.0 & 18.3 & .01 \\
\hline \multicolumn{6}{|l|}{$\mathrm{HbA}_{1 \mathrm{c}}$ category, $\%$} \\
\hline No current diabetes, $<5.7 \%$ & 53.2 & 32.5 & 41.1 & 53.2 & \multirow{5}{*}{.11} \\
\hline No current diabetes, $5.7 \%-6.4 \%$ & 20.2 & 28.5 & 21.2 & 26.5 & \\
\hline No current diabetes, $\geq 6.5 \%$ & 4.5 & 8.2 & 6.7 & 2.0 & \\
\hline Current diabetes, $<7.0 \%$ & 13.7 & 13.6 & 12.4 & 13.0 & \\
\hline Current diabetes, $\geq 7.0 \%$ & 8.4 & 17.2 & 18.6 & 5.4 & \\
\hline Had diabetes screening in past 3 years ${ }^{d}$ & 67.1 & 77.3 & 59.2 & 72.6 & .11 \\
\hline No health insurance, $\%$ & 12.5 & 14.6 & 34.8 & 17.0 & $<.001$ \\
\hline No routine health care location, $\%$ & 9.2 & 3.4 & 20.0 & 12.2 & .009 \\
\hline \multicolumn{6}{|l|}{ Education, $\%$} \\
\hline$<$ High school diploma & 12.2 & 16.6 & 45.3 & 5.3 & \multirow{4}{*}{$<.001$} \\
\hline High school diploma or equivalent & 20.0 & 19.4 & 19.3 & 15.4 & \\
\hline Some college & 39.4 & 40.6 & 26.7 & 27.9 & \\
\hline College graduate or higher & 28.3 & 23.4 & 8.8 & 51.4 & \\
\hline Income-to-poverty ratio ${ }^{\mathrm{e}}$ & $3.1(0.1)$ & $2.3(0.2)$ & $1.8(0.1)$ & $3.4(0.2)$ & $<.001$ \\
\hline
\end{tabular}

Abbreviations: $\mathrm{HbA}_{1 \mathrm{c}}$, glycated hemoglobin $\mathrm{A}_{1 \mathrm{c}}$

${ }^{a}$ All values are weighted mean (standard error) for continuous variables and weighted percentage for categorical or binary variables, unless otherwise indicated. Unweighted sample size ranged from 630-765 because of missing data.

${ }^{\mathrm{b}}$ Comprises women in all other racial/ethnic categories indicated in NHANES as well as women who indicated multiple race/ethnicities.

${ }^{c}$ Analysis of variance used to determine $P$ values for continuous values and $x^{2}$ tests for categorical values.

${ }^{d}$ Calculated only among those without diabetes at the time of NHANES data collection (unweighted $n=534$ ).

${ }^{\mathrm{e}}$ Income-to-poverty ratio was quantified by dividing family income by the poverty threshold determined by the US Department of Health and Human Services, specific to family size; a poverty-income ratio below 1 indicates that the family is below the poverty threshold.

The opinions expressed by authors contributing to this journal do not necessarily reflect the opinions of the U.S. Department of Health and Human Services, the Public Health Service, the Centers for Disease Control and Prevention, or the authors' affiliated institutions. 
Table 2. Racial/Ethnic Differences Among Women Aged $\geq 18$ Years in Association With $\mathrm{HbA}_{1 \mathrm{c}}$, National Health and Nutrition Examination Survey (NHANES), 2007-2016

\begin{tabular}{|c|c|c|c|c|}
\hline \multirow[b]{2}{*}{ Characteristic } & \multicolumn{2}{|c|}{$\operatorname{RRR}(95 \% \mathrm{CI})[P]$} & \multicolumn{2}{|c|}{$\mathrm{HbA}_{1 \mathrm{c}}, \beta(95 \% \mathrm{Cl})[P]$} \\
\hline & $\begin{array}{c}\mathrm{HbA}_{1 \mathrm{c}} 5.7 \%-6.4 \%^{\mathrm{a}} \\
\text { (Unweighted } \mathrm{n}=534 \text { ) }\end{array}$ & $\begin{array}{c}\mathrm{HbA}_{1 c} \geq 6.5 \%{ }^{\mathrm{a}} \\
\text { (Unweighted } \mathrm{n}=534 \text { ) }\end{array}$ & $\begin{array}{c}\text { No Diabetes } \\
\text { (Unweighted } n=534 \text { ) }\end{array}$ & $\begin{array}{c}\text { Diabetes } \\
\text { (Unweighted } n=231 \text { ) }\end{array}$ \\
\hline \multicolumn{5}{|l|}{ Model 1: Unadjusted } \\
\hline Non-Hispanic white & $1.00[$ Reference] & 1.00 [Reference] & 1.00 [Reference] & 1.00 [Reference] \\
\hline Non-Hispanic black & 2.31 (1.30 to 4.13$)$ [.005] & 2.98 (1.01 to 8.79) [.05] & 0.31 (0.07 to 0.56 ) [.01] & 1.12 (0.19 to 2.05$)[.02]$ \\
\hline Hispanic & 1.36 (0.82 to 2.26$)[.22]$ & 1.94 (0.80 to 4.68$)$ [.14] & 0.24 (0.05 to 0.43 ) [.01] & 0.90 (0.26 to 1.54$)[.006]$ \\
\hline Other & 1.32 (0.49 to 3.54) [.58] & 0.44 (0.11 to 1.86$)$ [.26] & $0.06(-0.10$ to 0.21$)[.45]$ & $-0.30(-0.83$ to 0.23$)[.26]$ \\
\hline
\end{tabular}

Model 2: Adjusted for age, education, marital status, and health insurance status

\begin{tabular}{|l|c|c|c|c|}
\hline Non-Hispanic white & 1.00 [Reference] & 1.00 [Reference] & 1.00 [Reference] & 1.00 [Reference] \\
\hline Non-Hispanic black & $3.46(1.77$ to 6.78$)[<.001]$ & $4.94(1.53$ to 15.96$)[.008]$ & $0.40(0.15$ to 0.65$)[.002]$ & $1.30(0.46$ to 2.14$)[.003]$ \\
\hline Hispanic & $1.98(1.07$ to 3.67$)[.03]$ & $3.49(1.57$ to 8.04$)[.004]$ & $0.34(0.14$ to 0.54$)[.001]$ & $1.17(0.55$ to 1.78$)[<.001]$ \\
\hline Other & $1.97(0.70$ to 5.50$)[.19]$ & $0.61(0.12$ to 3.23) [.56] & $0.14(-0.01$ to 0.29) [.06] & $-0.29(-0.84$ to 0.27) [.31] \\
\hline
\end{tabular}

Model 3: Model 2 + body mass index, waist circumference, hypertension status, and high-density lipoprotein cholesterol

\begin{tabular}{|c|c|c|c|c|}
\hline Non-Hispanic white & $1.00[$ Reference] & $1.00[$ Reference] & $1.00[$ Reference] & 1.00 [Reference] \\
\hline Non-Hispanic black & 3.35 (1.60 to 7.04$)[.002]$ & $5.24(1.39$ to 19.70$)[.02]$ & 0.34 (0.11 to 0.57) [.004] & $1.32(0.48$ to 2.15$)[.002]$ \\
\hline Hispanic & $2.20(1.17$ to 4.17$)[.02]$ & $6.72(2.51$ to 17.98$)[<.001]$ & $0.34(0.15$ to 0.53$)[.001]$ & 1.31 (0.71 to 1.91$) ;[<.001]$ \\
\hline Other & 2.09 (0.83 to 5.30$)[.12]$ & $0.96(0.08$ to 11.18$)[.97]$ & $0.14(0.01$ to 0.27$)[.03]$ & $-0.15(-0.67$ to 0.37$)[.57]$ \\
\hline
\end{tabular}

Abbreviations: $\mathrm{Cl}$, confidence interval; $\mathrm{HbA}_{1 c}$, glycated hemoglobin $\mathrm{A}_{1 c}$; RRR, relative risk ratio.

${ }^{a}$ Among women aged $\geq 18$ years who had complete information on covariates and who did not self-report a diagnosis of diabetes by a health care provider or insulin use at the time of the NHANES examination. Multinomial regression models were used to assess undiagnosed diabetes $\left(\mathrm{HbA}_{1 \mathrm{c}} \geq 6.5 \%\right), \operatorname{prediabetes}\left(\mathrm{HbA} \mathrm{A}_{1 \mathrm{c}}\right.$, $5.7 \%-6.4 \%)$, and no diabetes $\left(\mathrm{HbA}_{1 \mathrm{c}}<5.7 \%\right)$. No diabetes is reference group (or base outcome).

\footnotetext{
The opinions expressed by authors contributing to this journal do not necessarily reflect the opinions of the U.S. Department of Health and Human Services, the Public Health Service, the Centers for Disease Control and Prevention, or the authors' affiliated institutions.
} 
Table 3. Racial/Ethnic Differences in the Development of Type 2 Diabetes Among Women Aged $\geq 18$ Years With a History of Gestational Diabetes Mellitus ( $\mathrm{N}=$ 759), National Health and Nutrition Examination Survey, 2007-2016

\begin{tabular}{|c|c|c|c|c|}
\hline \multirow[b]{2}{*}{ Model } & \multirow[b]{2}{*}{ Non-Hispanic White } & \multicolumn{3}{|c|}{ Hazard Ratio (95\% Confidence Interval) $[P$ Value $]$} \\
\hline & & Non-Hispanic Black & Hispanic & Other \\
\hline Model $1^{\mathrm{b}}$ & 1.00 [Reference] & $1.95(1.32-2.88)[.001]$ & $2.17(1.44-3.25)[<.001]$ & $1.18(0.56-2.51)[.66]$ \\
\hline Model $2^{c}$ & 1.00 [Reference] & $1.71(1.13-2.58)[.01]$ & $1.68(1.09-2.60)[.02]$ & $1.57(0.80-3.09)[.19]$ \\
\hline Model $3^{d}$ & 1.00 [Reference] & $1.63(1.11-2.39)[.01]$ & $2.22(1.47-3.35)[<.001]$ & $2.08(1.01-4.28)[.047]$ \\
\hline
\end{tabular}

${ }^{a}$ Of 765 women aged $\geq 18$ years with a history of gestational diabetes mellitus, 6 did not have data on age at diabetes diagnosis.

${ }^{\mathrm{b}}$ Unadjusted.

${ }^{\mathrm{c}}$ Adjusted for age, education, marital status, and health insurance status.

${ }^{\mathrm{d}}$ Model 2 + body mass index, waist circumference, hypertension status, and high-density lipoprotein cholesterol. 
Table 4. Racial/Ethnic Differences in Screening for Diabetes Among Women Aged $\geq 18$ Years Without Current Diabetes $(n=496)$, National Health and Nutrition Examination Survey, 2007-2016

\begin{tabular}{|l|c|c|c|c|}
\hline \multirow{2}{*}{ Model } & \multirow{2}{*}{ Odds Ratio (95\% Confidence Interval) $[P$ Value] } \\
\cline { 3 - 5 } & Non-Hispanic White & Non-Hispanic Black & \multicolumn{2}{|c|}{ Hispanic } \\
\hline Unadjusted & 1.00 [Reference] & $1.89(0.98-3.22)[.06]$ & $0.66(0.40-1.07)[.09]$ & $1.16(0.55-2.48)[.69]$ \\
\hline + Income-to-poverty ratio ${ }^{\text {b }}$ & 1.00 [Reference] & $2.22(1.22-4.03)[.009]$ & $0.91(0.53-1.56)[.73]$ & $1.11(0.52-2.35)[.78]$ \\
\hline + Routine health care location & 1.00 [Reference] & $2.07(1.29-3.81)[.02]$ & $1.01(0.58-1.78)[.96]$ & $1.10(0.51-2.38)[.24]$ \\
\hline
\end{tabular}

${ }^{a}$ Among 534 women aged $\geq 18$ years with no diabetes, 38 were missing information on income-to-poverty ratio and routine health care location.

${ }^{b}$ Income-to-poverty ratio was quantified by dividing family income by the poverty threshold determined by the US Department of Health and Human Services, specific to family size; a poverty-income ratio below 1 indicates that the family is below the poverty threshold. 